\title{
An Entropy-Based Approach for Generating Multi-dimensional Sequential Patterns
}

\author{
Chang-Hwan Lee \\ Department of Information and Communications, \\ DongGuk University, \\ Seoul, Korea 100-715 \\ chlee@dgu.ac.kr
}

\begin{abstract}
This paper proposes a new method for generating multidimensional sequential patterns. While the current sequential pattern methods are generating patterns within a single attribute, the proposed method is able to detect them among different attributes. We employ an information theoretic method for generating multi-dimensional sequential patterns with the use of Hellinger entropy measure. A number of theorems are proposed to reduce the computational complexity of the sequential pattern systems. The proposed method is tested on some synthesized transaction databases.
\end{abstract}

\section{Introduction}

Among many techniques in data mining, sequential pattern is a technique which can discover more meaningful information by considering time attribute, together with other traditional attributes. Sequential patterns can be widely used in many different applications, such as mining banking patterns from bank accounts, and predicting certain kind of disease from history of symptoms.

Almost all of the current methods for mining sequential patterns are based on the Apriori algorithm 11, SPIRIT [6, FreeSpan [4, PrefixSpan [5], SPADE [9], CloSpan [8], and TSP [7. After that, a series of Apriori-like algorithms have been proposed. However, one of the limitations of the current sequential pattern algorithms is that they mine only one dimension. They only consider one attribute, and thus can not detect sequential patterns hiding across different attributes.

On the other hand, multi-dimensional sequential pattern mining attempts to find sequential patterns across several dimensions of attribute. By incorporating the additional attributes, the sequential patterns found are richer and more informative to the user. The aim of this multi-dimensional sequential pattern mining is to get more interesting sequential patterns with different dimensional attributes.

However, there is very little study on mining sequential patterns in multidimensional circumstances(e.g. UniSeq [3] ).

In this paper, we propose a new paradigm for generating multi-dimensional sequential patterns. We use an entropy function, called Hellinger measure, as an 
underlying tool for developing multi-dimensional sequential patterns. Therefore this method could provide more theoretic background in sequential pattern generation. Also, we replaced the traditional measures of sequential patterns(like support and confidence) by more sophisticated, information-theoretic measure. The proposed method could calculate the significance of each sequential pattern(called $H$ measure) as a numeric value, and those sequential patterns are given in a sorted order. The $H$ measure can be interpreted as the importance or significance of sequential patterns.

\section{Problem Description}

The format of sequential patterns generated in this paper is as follows:

$$
A=a \wedge B=b \wedge \cdots \rightarrow T=t \text { with } \alpha, \beta \text {, and } H
$$

where $A, B$ and $T$ are attributes with $a, b$ and $t$ being values in their respective discrete alphabets. We restrict the right-hand expression to being a single value assignment expression while the left-hand side may be a conjunction of such expressions. The semantics of above format is that if a person does an action(e.g., purchase) based on the condition(left-hand side) of above pattern at a given time, then he/she will later do an action described in right-hand side with high possibility $H$. Each sequential pattern comes with three numeric values such as $\alpha, \beta$, and $H$. The $\alpha, \beta$, and $H$ represent the information content, the generality, and the significance of sequential pattern, respectively. The interpretation of these numeric terms will be explained in the following section. The final sequential patterns generated from the database are sorted based on the $H$ value.

Since our sequential pattern method handles multi-dimensional databases, the format of database is different from the format used by traditional sequential pattern methods. Each transaction of database is associated with different attributes for multi-dimensional sequential patterns mining.

The transaction database is in its first normal form(each attribute, including the items, contains only one value). The database consists of a set of tuples

$$
<\text { cid }, \text { tid }, a_{1}, a_{2}, \ldots, a_{n}, c>
$$

where $c i d$ is an identification of the customer and tid the time. Let $a_{1}, a_{2}, \ldots, a_{n}$ denote the multi-dimensional attributes with respect to the customer, product, or transaction, and $c$ means the item bought by the customer cid. In case multiple items are purchased together, each of them is represented in different tuples with the same $c i d$ and $t i d$. In addition, the entire transaction database is sorted based primarily on customer-id(cid) and secondly on transaction-time $($ tid $)$.

\section{Information Contents of Sequential Patterns}

The basic idea of sequential pattern generation in this paper starts with the assumption that the value assignments in the left hand side of each sequential pattern affects the probability distribution of the right-hand side(target attribute). 
Intuitively speaking, if a certain value assignment has significantly changed the probability distribution of the target, it is clear that the given value assignment plays an important role in determining the class values of the target attribute. Therefore, it is a natural definition, in this paper, that the significance of a sequential pattern is interpreted as the degree of dissimilarity between a priori probability distribution and a posteriori probability distribution of the target attribute.

In this paper, this dissimilarity is defined as instantaneous information, which is the information content of the sequential pattern given that the left-hand side happens. The critical part now is how to define or select a proper measure which can correctly measure the instantaneous information.

We employ an entropy function, called Hellinger measure, as a tool for defining the information content of sequential pattern rules. The Hellinger measure was originally introduced by Beran [2], and is defined as

$$
\sqrt{\sum_{i}\left(\sqrt{p\left(t_{i}\right)}-\sqrt{p\left(t_{i} \mid a\right)}\right)^{2}}
$$

where $t_{i}$ denotes the value of attribute $T$. It becomes zero if and only if both a priori and a posteriori distributions are identical, and ranges from 0 to 1 . Unlike other information measures, this measure is applicable to every possible case of probability distributions. It can be interpreted as a distance measure where distance corresponds to the amount of divergence between a priori and a posteriori distribution. Therefore, we employ Hellinger measure as a measure of divergence, which will be used as the information amount of sequential patterns.

\section{Contents of $\boldsymbol{H}$ Measure}

In terms of the probabilistic sequential pattern rules, let us interpret the event $A=a$ as the target concept to be learned and the event(possibly conjunctive) $B=b$ as the hypothesis describing this concept. The information content of the sequential pattern rule is defined as

$$
[\sqrt{P(a \mid b)}-\sqrt{P(a)}]^{2}+[\sqrt{1-P(a \mid b)}-\sqrt{1-P(a)}]^{2}
$$

where $P(a \mid b)$ means the conditional probability of $A=a$ under the condition $B=b$ has happened beforehand. Notice that Equation (2) has a different form of definition from that of Equation (11). In sequential pattern generation, one particular value of class attribute appears in the right hand side of the pattern, and thus the probabilities for all other values are included in $1-P(a)$. In addition, we squared the original form of Hellinger measure because, by squaring the original form of Hellinger measure, we could derive a boundary of the Hellinger measure, which allows us to reduce drastically the search space of possible sequential pattern rules.

Another criteria we have to consider is the generality of the sequential patterns. The generality is similar to the support in Apriori-like methods. The basic 
idea behind generality is that the more often left-hand side occurs for a sequential pattern, the more useful the pattern becomes. In this paper, we use $\sqrt{P(b)}$ to represent the probability that the sequential pattern will occur and, as such, can be interpreted as the measure of sequential pattern generality. The reason for using the square root form of the original probability is that, by using the square root form, we could derive some boundaries of $H$ measure.

As a result, by multiplying the generality with the information content of the sequential pattern rules, we have the following term

$$
\sqrt{P(b)}\left[(\sqrt{P(a \mid b)}-\sqrt{P(a)})^{2}+(\sqrt{1-P(a \mid b)}-\sqrt{1-P(a)})^{2}\right]
$$

which possesses a direct interpretation as a multiplicative measure of the generality and information content of a given sequential pattern rule. In this paper, we call above multiplicative term as $H$ measure of sequential patterns.

\section{Sequential Pattern Generation}

We will now define the algorithm and discuss its basic ideas. The algorithm takes time-related database in the form of discrete attribute vectors and generates a set of $K$ sequential patterns, where $K$ is a user-defined parameter. The set of generated sequential patterns are the $K$ most informative(significant) sequential patterns from the database as defined by the $H$ measure.

The algorithm employs branch-and-bound with depth-first search over possible left-hand sides. The algorithm first generates all possible cases of first-order sequential patterns. The first-order sequential patterns are sequential patterns that have single value assignment in left-hand side, described as follows,

$$
B_{i}=b_{i j} \rightarrow A=a_{k}
$$

where $B_{i}, B_{i j}$, and $A_{k}$ represent $i$-th attribute, the value of $i$-th attribute, and the target value, respectively.

The algorithm proceeds then calculating the $H$ measures of each first-order sequential patterns, finding $K$ most informative sequential patterns in terms of $H$ measure, and then placing these $K$ sequential patterns in an ordered list, called BEST. The smallest $H$ measure, that of the $K$ th element of BEST, is then defined as the running minimum $H_{*}$. The critical part of the algorithm is the specialization criterion since it determines how much of the exponentially large hypothesis space actually needs to be explored by the algorithm.

From that point onwards, new patterns which are candidates for inclusion in the sequential pattern set have their $H$ measure compared with $H_{*}$. If greater than $H_{*}$, they are inserted in the list and the $K$ th sequential pattern is deleted. And $H_{*}$ is updated with the value of the $H$ measure of whatever sequential pattern is now $K$ th on the list. The algorithm systematically tries to specialize all first-order sequential patterns and terminates when it has determined that no more sequential patterns exist which can be specialized to achieve a higher $H$ measure than $H_{*}$. 
Figure 1 describes the pseudo code of decision whether to continue specializing or to back-up on the depth-first search. The $H$ measure of each sequential pattern can be considered as the weight of the sequential pattern.

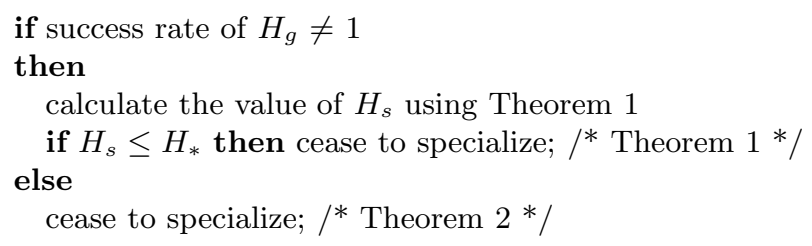

Fig. 1. Algorithm for specialization

\section{Characteristics of $\boldsymbol{H}$ Measure}

The characteristic of the specialization behavior is critical to the performance of the algorithm. Therefore, it is important to derive some quantitative bounds on the nature of specialization, which can be used to improve computational performance.

Specialization is the process by which we try to increase a sequential pattern's information content by adding an extra condition to the pattern's left-hand side. The consequent necessary decrease in generality of the sequential pattern should be less than an increase in the information content to the extent that the overall $H$ measure is increased. We will examine specialization, using the $H$ measure as the definition of sequential pattern goodness, with $\sqrt{p(a)}$ corresponding to generality and Equation (2) corresponding to information content. If we define $H_{s}$ and $H_{g}$ as the $H$ measures of the specialized and general sequential patterns, respectively, is it possible to find a bound of $H_{s}$ in terms of $H_{g}$ ?

Suppose we have a sequential pattern

$$
B=b \rightarrow A=a .
$$

We would like to specialize this sequential pattern by adding a condition $C=c$ so that we have a specialized sequential pattern

$$
B=b \wedge C=c \rightarrow A=a .
$$

For the sake of illustration, sequential patterns in formula (3) and (4) are denoted as $R_{g}$ and $R_{s}$, respectively. In this paper, we deal with a sequential pattern which contains only one condition and try to specialize it. More general cases which have more than one condition in the left hand side can be easily understood. Suppose $H_{g}$ and $H_{s}$ are the $H$ measures of the sequential patterns $R_{g}$ and $R_{s}$, respectively. Our goal is to answer the question "Can we describe the bound of $H_{s}$ in terms of $H_{g}$ ?" In other words, is it possible to estimate the maximum value of $H_{s}$ without knowing any information about attribute $C$ ? The motivation for bounding $H_{s}$ in this manner is two-folds. Firstly, it produces some theoretical insight into specialization, while secondly, the bound can be used by the sequential pattern algorithm to search the search space(hypothesis space) efficiently. 
Consider that we are given a general sequential pattern whose $H$ measure, $H_{g}$, is defined as

$$
H_{g}=\sqrt{P(b)}[2-2 \sqrt{P(a \mid b) P(a)}-2 \sqrt{(1-P(a \mid b))(1-P(a))}]
$$

We try to calculate the bound of

$$
\begin{aligned}
H_{s}= & \sqrt{P(c \mid b)} \sqrt{P(b)}[2-2 \sqrt{P(a \mid b c) P(a)}- \\
& 2 \sqrt{(1-P(a \mid b c))(1-P(a))}]
\end{aligned}
$$

Given no information about $C$, we can state the following results.

Theorem 1. If the $H$ measure of a specialized pattern satisfies the following boundary:

$$
\begin{array}{r}
H_{s} \leq \max \{\sqrt{P(a \mid b)} \sqrt{P(b)}[2 \sqrt{m}-2 \sqrt{P(a)}], \\
2 \sqrt{P(b)}-\sqrt{1-P(a \mid b)} \sqrt{P(b)}[2 \sqrt{P(a)}+2 \sqrt{1-P(a)}]\}
\end{array}
$$

where $m$ represents the number of class in the target attribute, the general pattern discontinues specializing.

Proof is omitted due to space limit. As a special case of Theorem 1, if the success rate, conditional probability $(P(a \mid b))$, of general pattern becomes 1 , the $H$ measure of the specialized pattern is always less than or equal to that of general pattern.

Theorem 2. If the conditional probability $(P(a \mid b))$ of general pattern is $1, H$ measure of specialized pattern cannot be greater than that of general pattern. Therefore, the general pattern discontinues specializing.

Proof is omitted due to space limit. As a consequence of these theorems we note that since the bound of specialized sequential pattern is achievable without further information about $C$, we can decide in advance that the specialized sequential pattern cannot be improved with respect to $H$ Measure. The logical consequence of this statement is that it precludes using the bound to discontinue specializing based on the value of $H_{g}$ alone. In particular, if the bound is less than the information content of the worst sequential pattern, then specialization cannot possibly find any better sequential pattern. This principle will be the basis for restricting the search space of the system.

\section{Experimental Results}

In order to test the functionality of the algorithm proposed in this paper, we assumed an artificial transaction database with 14 attributes, and synthesized two sets of artificial databases. The proposed algorithm was tested on two synthetic databases. Each database contains 20,000 records, and data values are generated 
Table 1. Sequential patterns using database I

\begin{tabular}{|l|c|c|}
\hline Sequential Patterns & Conf. & $H$ \\
\hline \hline Price $=20-29 \rightarrow$ Item $=$ P07 & 0.13137 & 0.00023 \\
Gender $=$ male \& Item $=$ P06 $\rightarrow$ Item=P02 & 0.11015 & 0.00021 \\
Qty $=1 \rightarrow$ Item $=$ P09 & 0.11800 & 0.00021 \\
Item $=$ P09 $\rightarrow$ Item =P01 & 0.11067 & 0.00019 \\
SaleorNot=sale $\rightarrow$ Item =P00 & 0.11207 & 0.00017 \\
Age $=20-29 \&$ Qty $=$ over $5 \rightarrow$ Item $=$ P03 & 0.10592 & 0.00015 \\
Price $=30-39 \&$ Qty $=1 \rightarrow$ Item $=$ P02 & 0.10559 & 0.00015 \\
\hline
\end{tabular}

using random numbers. For each data set, the entire data set is read and then 100 most informative sequential patterns were generated.

The topmost 6 sequential patterns from the first database is shown in Table 1. For each pattern in Table 1, its corresponding values for confidence(Conf.) and $H$ measure are shown, and the resulting patterns are sorted based on their $H$ measure values. The confidence means the number of transactions satisfying both left-hand side and right-hand side of the pattern divided by the number of transactions satisfying left-hand side only.

The topmost pattern in Table 1 means that customers who purchased items (whatever the items are) of which price are between 20-29 later purchase item P07. This type of patterns can not be acquired from traditional sequential pattern methods. The 4 th pattern shows a sequential pattern equivalent to the one generated from Apriori-like method. It illustrates that the functionality of our method includes that of traditional sequential pattern methods.

The second database also contains 20,000 records, and data values are generated using random numbers. However, in the second database, we assumed that there are a number of sequential patterns hiding in the real world, and the database is generated based on those sequential patterns. The sequential patterns we have assumed are as follows.

- Color $=$ white \& Qty $=1 \rightarrow$ Item $=$ P05

- Region= city \& Item $=10-19 \rightarrow$ Item $=$ P08

The goal of this experiment is to verify whether the proposed algorithm is able to detect these sequential patterns hidden in the database. For the second

Table 2. Sequential patterns using database II

\begin{tabular}{|l|c|c|}
\hline Sequential Patterns & Conf. & $H$ \\
\hline \hline Item $=1 \rightarrow$ Item $=$ P07 & 0.17834 & 0.000181 \\
Color $=$ white \& Qty $=1 \rightarrow$ Item $=$ P05 & 0.15481 & 0.000103 \\
Price $=10-19 \rightarrow$ Item $=$ P03 & 0.13250 & 0.000065 \\
Price $=30-39 \rightarrow$ Item $=$ P09 & 0.14624 & 0.000051 \\
Region $=$ city \& Item $=10-19 \rightarrow$ Item=P08 & 0.11951 & 0.000040 \\
Color $=$ white \& Qty $=1 \rightarrow$ Item $=\mathrm{P} 08$ & 0.11440 & 0.000040 \\
\hline
\end{tabular}


experiment, the entire data set is read and then 100 most informative sequential patterns were generated. The topmost 6 sequential patterns from the second database is shown in Table 2, The sequential patterns we have assumed are generated from the system and shown in Table 2 as the 2nd and 5th pattern, respectively. We could also see many other multi-dimensional sequential patterns in Table 2. This experiment illustrates that our proposed algorithm is able to effectively detect the sequential patterns hidden within the database.

\section{Conclusion}

In this paper we have introduced a new method for generating multi-dimensional sequential patterns from transaction databases. We developed an information theoretic measure, called $H$ measure, which becomes the criteria for selecting and sorting inductive sequential patterns generated. The boundary of the $H$ measure is analyzed and two heuristics are developed to reduce the computational complexity of the system. In addition, missing values can be handled by considering them as separate categories. The algorithm is applied to some synthetic transaction databases. The resulting sequential patterns generated from the data sets show how the system detects the hidden multi-dimensional sequential patterns of data sets effectively.

\section{References}

1. R. Agrawal and R. Srikant, Mining sequential patterns, Int. Conf. on Data Engineering, 1995, pp. 3-14.

2. R. J. Beran, Minimum hellinger distances for parametric models, Ann. Statistics $\mathbf{5}$ (1977), 445-463.

3. J. Pei-K. Wang Q. Chen H. Pinto, J. Han and U. Dayal, Multi-dimensional sequential pattern mining, Int. Conf. on Information and Knowledge Management, 2001.

4. B. Mortazavi-Asl Q. Chen U. Dayal J. Han, J. Pei and M-C. Hsu, Freespan: Frequent pattern-projected sequential pattern mining, Int. Conf. Knowledge Discovery and Data Mining (KDD00), 2000.

5. B. Mortazavi-Asl H. Pinto Q. Chen U. Dayal J. Pei, J. Han and M.-C. Hsu, Prefixspan: Mining sequential patterns efficiently by prefix-projected pattern growth, Int. Conf. on Data Engineering, 2001.

6. R. Rastogi M. Garofalaskis and K. Shim, Spirit:sequential pattern mining with regular expression constraints, Int. Conf. on Very Large Databases, 1999.

7. Jiawei Han Petre Tzvetkov, Xifeng Yan, Tsp: Mining top- $k$ closed sequential patterns, Int. Conf. on Data Mining, 2003.

8. Ramin Afshar Xifeng Yan, Jiawei Han, Clospan: Mining closed sequential patterns in large databases, Int. Conf. on Data Mining, 2003.

9. M. J. Zaki, Spade: An efficient algorithm for mining frequent sequences, Machine Learning 42 (2001), 31-60. 